\title{
FLUORESCENT N-HETEROCYCLES VIA ONE-POT TANDEM REACTIONS
}

\author{
Rodica Dinica* ${ }^{1}$, Bianca Furdui ${ }^{1}$, Geta Cârâc ${ }^{1}$ and Constantin Stanciu ${ }^{1}$ \\ 1 "Dunarea de Jos" University, Galati, Chemistry Department, \\ Domneasca street, 47, Romania, E-mail: rodinica@ugal.ro
}

\begin{abstract}
Synthetic indolizines have attracted special attention in the past years mainly due to their manifold practical utilities. An improved preparation of bis-indolizines using microwave-assisted synthesis is described. The microwave-mediated three component reaction of acyl bromide, bipyridine and dipolarophyl is catalyzed by basic alumina to give corresponding bis-indolizines in excellent yields in a one -pot reaction. This strategy would provide access to fast synthesis of bis-indolizines which otherwise are accessible only through multistep synthesis.
\end{abstract}

Keywords: microwave, "one-pot” reaction, indolizine

\section{Introduction}

The conception of molecules with biologic properties is very interesting and opens a field of research important for molecular biology and pharmacology. Microwave irradiation is known to accelerate organic reactions and is a powerful tool in organic synthesis and in biocatalysis. The microwave-promoted solid phase heterogenous reaction is well-known as an environmentally benign reaction methodology that usually provides improved selectivity, enhanced reaction rates, cleaner products and manipulative simplicity [1-10]. The objective of the present study was to establish the viability of a three-component reaction (3-CR) involving a 1,3-dipolar cycloaddition reaction between an in situ generated dipole (from $\omega$-bromacetophenones and 4,4'-bipyridine) and ethyl propiolate, using a fast and facile reaction strategies that involve microwave energy as unconventional energy source. This strategy would provide access to fast one-pot synthesis of bis-indolizines which otherwise are accessible only through multistep synthesis [14-16]. 1,3-Dipolar cycloaddition has been widely used for the synthesis of heterocyclic compounds because of its utility for creating two sets of bonds in a single operation and because stereoselectivity and regioselectivity are easily predictable [11]. Among a number of 1,3-dipoles, azomethine ylides are useful intermediates for the synthesis of various fivemembered nitrogen-containing heterocycles. They belong to the class of 1,3-dipoles of the allyl type with a cationic nitrogen atom and react with several dipolarophiles to give pyrrolidine derivatives, respectively [12$13]$.

\section{Results and discussion}

We report here the synthesis of novel nitrogen heterocycles, with fluorescent properties. These compounds are easy prepared using microwave-assisted synthesis. The microwave-mediated three component reaction of phenacyl bromide, bipyridine and dipolarophyl is catalyzed by basic alumina to give corresponding bis-indolizines in excellent yields in a one-pot reaction.

Previously, we have studied the synthesis of this type of compounds using multistep reactions. Substituted indolizines are very attractive heterocyclic units, as a number of representatives of this class and, especially their partially or completely reduced analogues, indolizidine alkaloids and related natural compounds which exhibit important biological properties. As a part of our program on developing efficient methods for differently substituted indolizines, we were particularly interested in elaborating efficient approaches toward three-component reaction of these compounds which are very interested from the point of view fluorescent properties, certain of them being useful for securely marking articles, such as documents and security paper.

If successful, such a strategy would provide access to fast one-pot synthesis of cycloadducts which otherwise are accessible only through multistep synthesis. The first successful examples of the application of this approach are described here in (Scheme 1). 


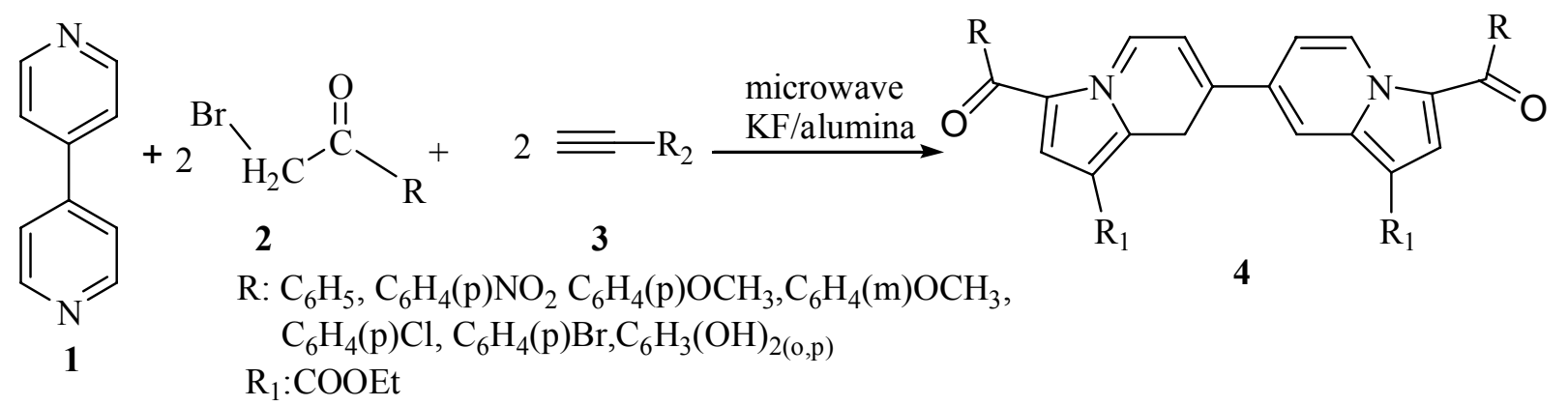

Scheme 1. One-pot synthesis of bis-indolizines

The design of multicomponent reactions (MCR) is an important field of research from the point of view of combinatorial chemistry. Being a one-pot reaction, generally multicomponent reactions afford good yields.

The indolizines constitute the core structure of many naturally occurring alkaloids (-)slaframine, (-)dendroprimine, indalozin and coniceine. The synthesis of biologically active indolizines continues to attract the attention of organic chemists.

The indolizines are most commonly synthesized by sequential $N$-quarternization and intramolecular cyclocondensation reactions or the cycloaddition reaction of $N$-acyl/alkyl bipyridinium salts. These strategies, however, involved multistep synthesis employing two-component reactions and do not represent the goal of an ideal synthesis.

Bis-indolizinic compounds were prepared, by classical reaction, according the retrosynthetic strategy based on cicloaddition reaction (Scheme 2) [14-15].

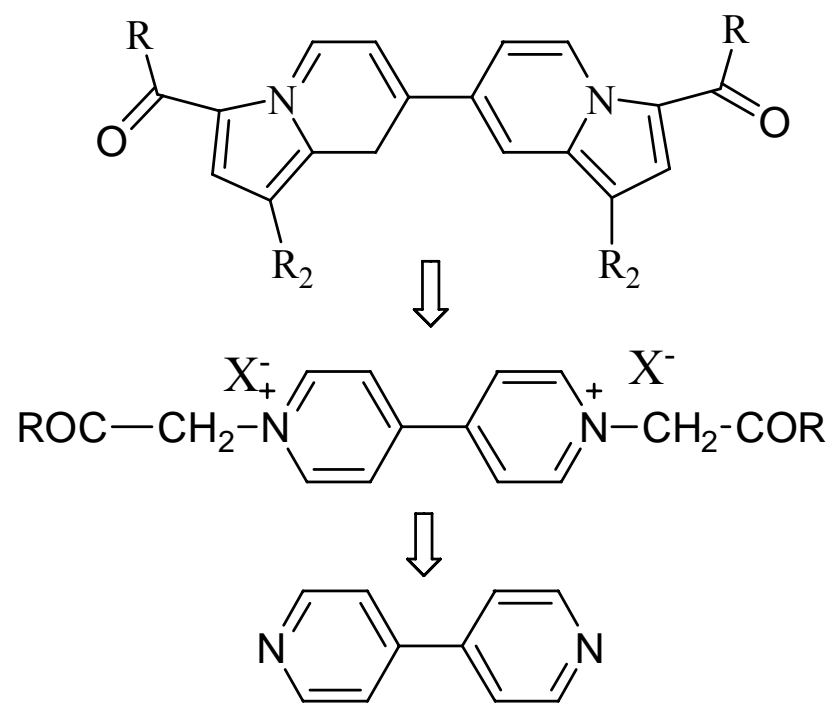

\section{Scheme 2. Retrosynthetic strategy for obtaining of bis-indolizines}

It was reasoned that basic alumina can be used as a basic catalyst under microwave energy for in situ dipole generation from the $N, N^{\prime}$-biphenacyl bipyridinium salt, which could participate in a [3+2] intramolecular cycloaddition reaction with acetylene dienophiles. Thus, when a three-component mixture of phenacyl bromide, 4,4'- bipyridine, and ethyl propiolate was thoroughly mixed in basic alumina and irradiated in a domestic oven for $10 \mathrm{~min}$, the reaction products was obtained in very good yields In the following table there are the results obtained in synthesis of bis-indolizines by classical reactions, in multistep way and one-pot synthesis. 
Bis-indolizine's yields by classical reactions, in multistep and one-pot synthesis

\begin{tabular}{|c|c|c|c|c|c|}
\hline \multirow{2}{*}{ Reaction type } & Solvent & $\begin{array}{c}\text { Activation } \\
\text { mode }\end{array}$ & $\begin{array}{c}\text { Time } \\
(\mathbf{m i n})\end{array}$ & $\begin{array}{c}\text { T } \\
\left({ }^{\circ} \mathbf{C}\right)\end{array}$ & $\begin{array}{c}\boldsymbol{\eta} \\
(\mathbf{\%})\end{array}$ \\
\hline \multirow{2}{*}{$\begin{array}{c}\text { Multistep reaction } \\
\text { (second step) }\end{array}$} & N-methylpirolidone & $\mathrm{MW}$ & 15 & 95 & $70-75$ \\
& & $\Delta$ & 300 & 50 & $60-65$ \\
\cline { 2 - 6 } & No solvent & $\mathrm{MW}$ & 15 & 95 & $67-70$ \\
& & $\Delta$ & 300 & 50 & $65-70$ \\
\hline One-pot reaction & N-methylpirolidone & $\mathrm{MW}$ & 15 & 95 & $72-75$ \\
& & $\Delta$ & 300 & 95 & $65-70$ \\
\cline { 2 - 7 } & No solvent & $\mathrm{MW}$ & 15 & 95 & $70-75$ \\
& & $\Delta$ & 300 & 95 & $70-75$ \\
\hline
\end{tabular}

The usefulness of this methodology lies in the fact that the three-component reactions are carried out rapidly under microwave-promoted environmentally benign, solvent-less conditions to give substituted indolizines in excellent yields. The catalytic effect of basic alumina was found more prominent in solid-phase 3-CRs than liquid-phase 3-CRs (Table 1 ). The reaction is compatible with substitutents such as aroyl groups and bipyridines. In conclusion, the methodology reported herein denotes a new class of 3-CR, which is expected to be a general route for the facile, one-pot combinatorial synthesis of a wide range of bis-indolizines.

\section{Experimental}

General Synthetic Procedure. 4,4'-bipyridine, 1, (1 mmole), phenacylbromide derivative, 2, (3 mmole), dipolarophyle,3, ( 3 mmole $)$, and basic alumina $(2 \mathrm{~g})$ were added in a $50 \mathrm{~mL}$ beaker. The mixture was stirred to obtain a free flowing powder, which was irradiated in a microwave oven at $300 \mathrm{~W}$ for an appropriate time (monitored by TLC). After cooling to room temperature, the product was extracted with chloroform $(3 \times 15 \mathrm{~mL})$. The product obtained after removal of solvent under reduced pressure was crystallized from a suitable solvent (EtOAc-petroleum; ether; $\mathrm{MeOH}$ ). The structure of the bis-indolizinic products, 4, was confirmed by spectral data and comparison with authentic samples prepared according to literature methods [14-16]. All reactions were carried out in a commercially available BPL BMO 800T multimode microwave reactor having a maximum power output of $800 \mathrm{~W}$ operating at $2450 \mathrm{MHz}$. Mass spectra were recorded using MSQ-Plus spectrometer. Melting points were recorded on a Metler Toledo melting point apparatus and are uncorrected.

\section{Conclusion}

A rapid, economic and environmentally friendly method has been developed for bis-indolizine synthesis using KF/basic alumina. The reagent system described here may be a good alternative to well known methods since the cycloaddition proceeds expeditiously with high yields under solvent-free conditions. These procedures are clearly dependent on the reaction medium and mechanisms involved.

Solvent-free organic synthesis offers a lot of advantages connected to safety, enhancement in reactivity and selectivity, cost saving, and energy and pollution prevention. When coupled with microwave irradiation, no thermal effects can be developed, thus allowing considerable improvements over classic procedures.

By this way, it can be improved the selectivity, enhanced the reaction rate and it can be obtained the cleaner products.

\section{Acknowledgment}

The authors thank the Romanian National Authority for financial research support (CNMP Nr.71/2007-2010).

\section{References}

[1]. Gundersen, L.L., Charnock, C., Negussie, A. H, Rise, F., Teklu, S., Eur. J. Pharmaceut. Sci., 2007, 30,26-35;

[2]. Hao, L., Zhiqiang, X., Shoujun, C., Koya, K., Ono, M., and Sun, L., Org. Process Res. Dev., 2007, 11 (2), 246 -250;

[3]. Landy, D.G. Surpateanu, G., Fourmentin, S., Blach, P., Decockand, P., Surpateanu, Gh., Internet Electronic Journal of Molecular Design, 2005, 545-555;

[4]. Qin, R. T. W., , Dahaen, B. N, Eur J Org Chem, 2006, 20:4658-4663;

[5]. Pessoa-Mahana, H., J. Kosche, et all, Heterocycles, 2008, 75, 8; 
[6]. Adam, D., Nature, 2003, 421, 571-572;

[7]. Satya, P., Nanda, P. and Gupta, R., Molecules 2003, 8, 374-380;

[8]. Kappe, C.O., Dallinger, D., Murphree, S.S., Practical Microwave Synthesis for Organic Chemists - Strategies, Instruments, and Protocols, Edition - 2009, Wiley-VCH;

[9]. Caddick, S., Tetrahedron, 1995, 51, 10403-10432;

[10]. Loupy, A., Petit, Hamelin, A. J., Texier-Boullet, F., Jacquault, P., Mathé, D, Synthesis, 1998, 1213-1234;

[11]. Huisgen, R. In 1,3-Dipolar Cycloaddition Chemistry; Padwa, A., Ed.; John Wiley \& Sons: New York, 1984; Vol. 1;

[12]. For reviews, see: (a) Lown, J. W. In 1,3-Dipolar Cycloaddition Chemistry; Padwa, A., Ed.; John Wiley \& Sons: New York, 1984; Vol. 1, p 653. (b) Vedejs, E.; West, F. G., Chem. Rev. 1986, 86, 941. (c) Tsuge, O.; Kanemasa, S. In Advances in Heterocyclic Chemistry; Katritzky, A. R., Ed.; Academic Press: San Diego, 1989; Vol. 45, p 231;

[13]. For a review, see: Grashey, R. In 1,3-Dipolar Cycloaddition Chemistry; Padwa, A. Ed.; John Wiley \& Sons: New York, 1984; Vol. 1, p 733;

[14]. Dinica, R., Pettinari, C., Heterocyclic Comm., 2001,07, 4, 381-386;

[15]. Dinica, R., Druta, I., Pettinari, C., Synlett, 2000, 222, 1013;

[16]. Furdui, B., Dinică, R., Druță, I., Demeunynck, M., Synthesis, 2006, 16, 2640-2642. 